Z. klin. Chem. u. klin. Biochem.

8. Jg., S. 579-581, November 1970

\title{
Kapillarblutentnahme mit Einweg-Mikrokapillaren zur Hämoglobin- und Glucosekonzentrationsbestimmung
}

\author{
Von P. Hilger, E. Henkel und A. Delbrück \\ Institut für Klinische Chemie, Abt. II, Zentrallaboratorium am Krankenbaus Oststadt der Mediqinischen Hochscbule \\ Hannover
}

(Eingegangen am 30. Juni 1970)

Es wird über heparinisierte Mikrokapillaren (20 $\mu l$ Volumen) zur Kapillarblutentnahme berichtet sowic über die Bestimmung der Blutglucose an dem gewonnenen Material mit Hilfe der o-Toluidin-Methode ohne Eisessig im Autoanalyzer.

The sampling of capillary blood with one-nvay microcapillaries for the determination of baemoglobin and glucose concentrations

Heparinized microcapillaries $(20 \mu l)$ are used for taking capillary blood. A microscale blood glucose determination using the o-toluidine method without acetic acid is adapted to the AutoAnalyzer system.

$\mathrm{Zu}$ den häufigsten Untersuchungsverfahren, die insbesondere auch im Nacht- und Notdienst geübt werden, gehören die Bestimmung des Hämoglobingehaltes und der Glucosekonzentration in Vollblut. Obwohl neben der Ermittlung dieser beiden Kenngrößen aus Kapillarblut in zunehmendem Maße Venenblut verwandt wird, besitzt die Kapillarblutentnahme für diese $Z$ wecke jedoch erhebliche Vorteile. Sie liegen vor allem in der einfachen Entnahmetechnik und in dem geringen Volumen der Blutprobe, die entnommen werden muß. Trotz dieser unzweifelhaften Vorteile wird das übliche Verfahren der Kapillarblutentnahme mit der 100- $\mu$-Blutglucose-Pipette bzw. 20- $\mu$-Hämoglobin-Pipette nicht allen zu stellenden Forderungen gerecht. Wesentliche Nachteile sind das relativ große Probevolumen von $100 \mu \mathrm{l}$, das exakt aus Kapillarblut aufzunehmen nur der Geübte in der Lage ist, die aufwendige Pipettenreinigung nach Dispensierung der Blutprobe in die Enteiweißungslösung und die aus hygienischen Gründen abzulehnende Benutzung des Ansaugeschlauches bei der Blutentnahme. Des weiteren zeigt sich immer wieder, daß Pipetten benutzt werden, welche an ihrer Spitze abgebrochen sind, wodurch - besonders bei der Hämoglobinbestimmung ins Gewicht fallend - ein Entfernen außen anhaftender Blutreste sehr erschwert wird. Hinzu kommt, daß die Technik der Aufnahme der Blutprobe durch Ansaugen insbes. bei der Blutglucosebestimmung, die das große Volumen erfordert, außerordentlich zeitaufwendig ist. Die dargelegten Gründe haben uns veranlaßt, nach einer einfacheren und schnelleren Entnahmemethode zu suchen, welche gute Präzision bei der Entnahme des Kapillarblutes auch durch ungeübte Kräfte ermöglicht. Ein solches System sollte den Gebrauch von Einweg-Material wie Einmallanzetten, Einmalpipetten und Einmalplastikgefäßen erlauben, ferner sollte bei möglichst kleinem Blutvolumen eine quantitative Entleerung der Pipette möglich sein und das Blut sollte in der Pipette nicht gerinnen können. Eine Verwendung des Ansaugeschlauches sollte wegen der damit verbundenen höheren Infektionsgefahr vermieden werden. Alle die aufgestellten Forderungen werden bei Verwendung von heparinisierten Mikrokapillaten definierten Volumens ( $\mathrm{Fa}$. Wilhelm Vogel, Gießen) erfüllt. Wir wählten ein Volumen von $20 \mu l$, das eine unveränderte Verwendung der Methode zur Hämoglobinbestimmung sowie die Anwendung zur Entnahme von Kapillarblut für die Blutglucosebestimmung erlaubt. Die Abmessung der Kapillare wurde bestimmt durch die Forderung nach einem möglichst günstigen Verhältnis Länge zu Durchmesser und durch die Höhe derEppendorf-Reaktionsgefäße (Netheler \& Hinz GmbH Hamburg), in denen die Kapillaren ausgeschüttelt werden. Zur Handhabung der Kapillaren dienen eigens hierfür gefertigte Scherenhalter.

\section{Material und Methoden}

Bestimmung der Präzision des Kapillarvolumens

Durch Auswaage mit Wasser bzw. Blutserum als visköser Lösung. Photometriscls mittels angefärbten Blutserums (Amidoschwarz).

\section{Hämoglobinbestimmung}

Blut-Entnahme

Nach Einstich der Lanzette und Verwerfen des ersten Bluttropfens füllt sich die Kapillare bei waagerechter Haltung schnell mit dem austretenden Blut. Entfernen anhaftender Blutreste an der Kapillaraußenseite mit einem Tupfer. Die Kapillare wird in ein vorbereitetes Gefäß mit $5 \mathrm{ml}$ DraBknsscher Lösung gegeben und gut umgeschüttelt.

Bestimmung des $\mathrm{Hämoglobingehaltes}$

Entsprechend den Empfehlungen des Internationalen Standardisierungsausschusses für Hämatologie $\operatorname{ICSH}(1,2)$. Für die Bestimmung der Präzision in der Serie wurde EDTA-Blut verwandt, das durch ständiges Rühren in Suspension gehalten wurde.

Bestimmung der Blutglucosekonzentration:

Blut-Entnahme

Einfließenlassen des Kapillarblutes wie oben.

Nach sorgfältiger Reinigung der Außenseite der Kapillare wird diese in ein vorbercitetes Reaktionsgefäß Eppcndorf gegeben, welches $0,5 \mathrm{ml} 50 \mathrm{~mm}$ Natriumfluoridlösung enthält. Es wird gut durchgeschüttelt. Die Enteiweißung erfolgt durch Zugabe 
von $0,1 \mathrm{ml}$ Perchlorsäure zu einer Endkonzentration von $0,33 \mathrm{M}$. Abtrennen des Sedimentes unter Verbleiben der Mikrokapillare im Reaktionsgefäß bci 18000 U/Min. für 2 Minuten in der Mikrozentrifuge Eppendorf (Netheler \& Hinz GmbH, Hamburg).

Bestimmung der Blutglucosekonzentration

mit dem o-Toluidin-Gljcolsäure-Reagenz (3) im Autoanalyzer.

Es werden in der Reihenfolge

Athylenglycol-Monomethyläther (Merck Nr. 854) $450 \mathrm{ml}$

Methanol (Merck Nr. 6009)

$50 \mathrm{ml}$

demin. Wasser

$50 \mathrm{ml}$

o-Toluidin (Merck Nr. 8310)

$100 \mathrm{ml}$

Glycolsäure (Merck Nr. 4104)

$120 \mathrm{~g}$

Äpfelsäure (Merck Nr. 382)

$120 \mathrm{~g}$

Thio-Harnstoff (Merck Nr. 7979)

zusammengegeben. Die Äpfelsäure darf erst nach vollständigem Lösen der Glycolsäure zugesetzt werden. Das Analyzer-Manifold ist aus dem Fließdiagramm (Abb. 1) ersichtlich. Neben Tygonpumpenschläuchen sind die Schlauchverbindungen durch Teflonschlauch ( $1 \mathrm{~mm}$ innerer $\varnothing$ ) hergestellt. Zwischen Heizbad und Kolorimeter ist eine Kühlschlange eingeschaltet, um das Reagenz auf Raumtemperatur abzukühlen und damit Undichtigkeiten der Verbindungsmuffen zur Durchflußküvette infolge Hitzeaus-

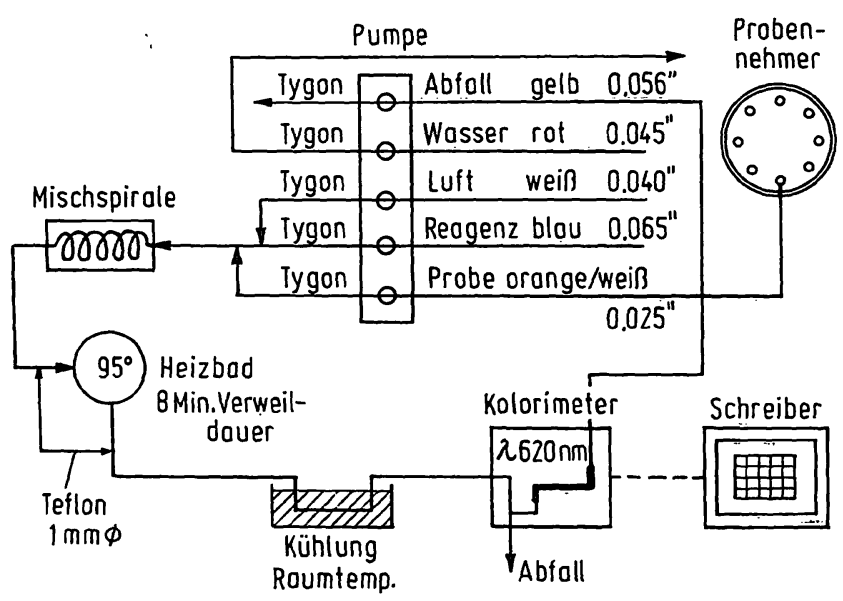

Abb. 1

Fließdiagramm zur Bestimmung der Glucose im Kapillarblut im Autoanalyzer (Mikromanifold, o-Toluidin-Methode mit Äpfel- und Glycolsäure in Äthylenglycolmonomethyläther)

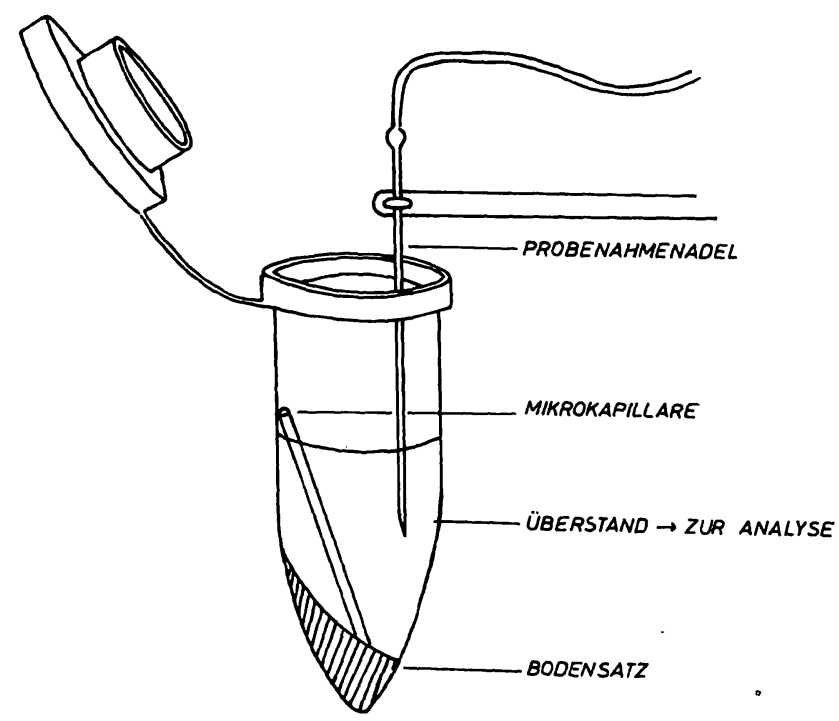

Abb. 2

Mikrokapillare und Reaktionsgefäß bei der Probenahme im Autoanalyzer

dehnung zu vermeiden. Die Messung erfolgt bei $620 \mathrm{~nm}$ im Kolorimeter und wird kontinuierlich auf dem Technicon-Schreiber aufgezeichnet. Die Probefrequenz beträgt 40/Min., die Zeitgeberscheibe am Probenehmer stellt ein Verhältnis von Waschund Probeansaugzeit von 1:1 ein. Kapillare und Sediment verbleiben im Probengefäß (Abb. 2).

\section{Enzymatische Blutglucosebestimmung}

entsprechend den Angaben von Barthelmar und Czok (4). Probevolumen $0,1 \mathrm{~m} l$ Perchlorsäureüberstand, Endvolumen $1 \mathrm{~m} l$.

\section{Ergebnisse und Diskussion}

Heparinisierte Mikrokapillaren definierten Volumens erwiesen sich den konventionellen Entnahmeverfahren überlegen hinsichtlich der Handhabung, des benötigten Zeitaufwandes und der Forderung nach Infektionsschutz im Laboratorium. Die verwandten Kapillaren besitzen eine hohe Präzision. Der gravimetrisch und photometrisch ermittelte Variationskoeffizient des Volumens beträgt $0,6-0,8 \%$ je nach Charge.

Die Präzision der Methode zur Hämoglobinbestimmung mit Mikrokapillaren hängt weitgehend von der Präzision ab, mit der die Volumina der DrabKINschen Lösung bzw. der Blutprobe bemessen werden. Mit einem Variationskoeffizienten von $0,6 \%$ erzielten wir bei der Blutentnahme mit Mikrokapillaten und Verwendung des Brand-Dispensers (Fa. Brand, Wertheim/Main) zur Bemessung der Drabkrnschen Lösung ein Ergebnis, das von dem der gravimetrischen Volumenbestimmung der Mikrokapillaren nicht abweicht.

Die Reduzierung des Blutvolumens von $100 \mu l$ auf $20 \mu l$ für die Bestimmung der Glucose in Kapillarblut erfordert für die Benutzung des Autoanalyzers Technicon die Anwendung einer Mikromethode. Zu diesem Zweck erwies sich uns die von HärTEL und Mitarbeitern (3) angegebene Methode zur Bestimmung der Blutglucosekonzentration mit $o$-Toluidin, welche anstelle von Eisessig Äpfel- und Glycolsäure in Äthylenglycol-Monomethyläther verwendet, wegen der relativ hohen Empfindlichkeit besonders geeignet. Durch Variation des Mischungsverhältnisses der Reaktionspartner (s. o.) gelang es, die Viskosität des Reaktionsgemisches so herabzusetzen, daß dieses Verfahren im Autoanalyzersystem, auch im Mikromaßstab, Verwendung finden kann. Bei der Prüfung der Präzision der BlutglucoseKonzentrationsbestimmung in der Serie fanden wir einen Variationskoeffizienten von 2,3\% $(n=78)$ für eine Glucosekonzentration von $109 \mathrm{mg} / 100 \mathrm{ml}$ und von $2,2 \%(n=100)$ für eine Konzentration von $294 \mathrm{mg} /$ $100 \mathrm{ml}$. Als Basis für den Vergleich der Ergebnisse beider Entnahmeverfahren (Mikrokapillare und Blutzuckerpipette) wurde die enzymatische Bestimmung der Glucosekonzentration mit Hexokinase-Glucose-6-phosphat-Dehydrogenase gewählt. Sowohl bei der Ermittlung der Glucosewerte mit der o-Toluidin-Methode wie der enzymatischen Bestimmung ergab sich eine ausgezeichnete Korrelation der Ergebnisse aus den beiden unterschiedlichen Entnahmeverfahren. Die Korrelationskoeffizienten betrugen 0,9977 ( $\mathrm{n}=24)$ bzw. $0,9982(n=22)(A b b .3,4)$. 
Aus Anlaß der hundertsten Wiederkehr des Jahres, in dem zum ersten Mal Margarine hergestellt wurde, hat das Margarine-Institut für gesunde Ernährung, Hamburg, eine

\title{
Studienförderung der
}

\section{Ernährungswissenschaften}

\author{
ins Leben gerufen.
}

Die Studienförderung umfaßt zehn Stipendien, jedes gewährt auf zwei Jahre. Der monatliche Stipendienbetrag ist DM 400.

Durch diese Stipendien sollen begabte Doktoranden an deutschen Hochschulen gefördert werden, die sich auf eine Promotion über

chemische, biochemische, physiologische, medizinische Aspekte der menschlichen Ernährung, insbesondere über die Rolle der Fette in Ernährung und Stoffwechsel, vorbereiten. Die Bewerber bedürfen der Empfehlung durch einen Hochschullehrer.

Über die Vergabe der Stipendien entscheidet ein Gutachterausschuß, der aus deutschen Hochschullehrern besteht. Ihm gehören gègenwärtig an:

$\begin{array}{ll}\text { Prof. Dr. W. Droese } & \text { Prof. Dr. G. Schettler } \\ \text { Prof. Dr. W. Heimann } & \text { Prof. Dr.Th.Wieland } \\ \text { Prof. Dr. J. Kühnau } & \text { Prof. Dr. V.Wolf } \\ \text { Prof. Dr. Dr. K. Lang } & \text { Prof. Dr. N. Zöllner }\end{array}$

Bewerbungen sind zu richten an den Beauftragten des Gutachterausschusses, Prof. Dr. Alfons Fricker, 7501 Grötzingen, Ringelberghohl 12 


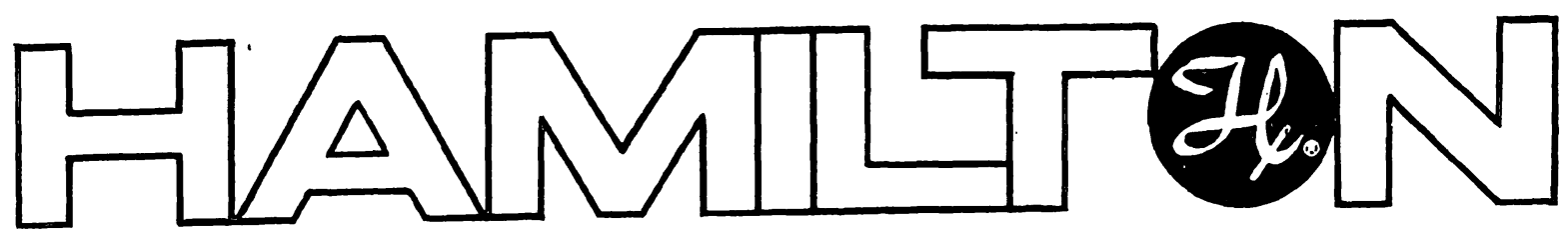

HAMILTON Präzisionsdosiervorrichtung für Flüssigkeiten.

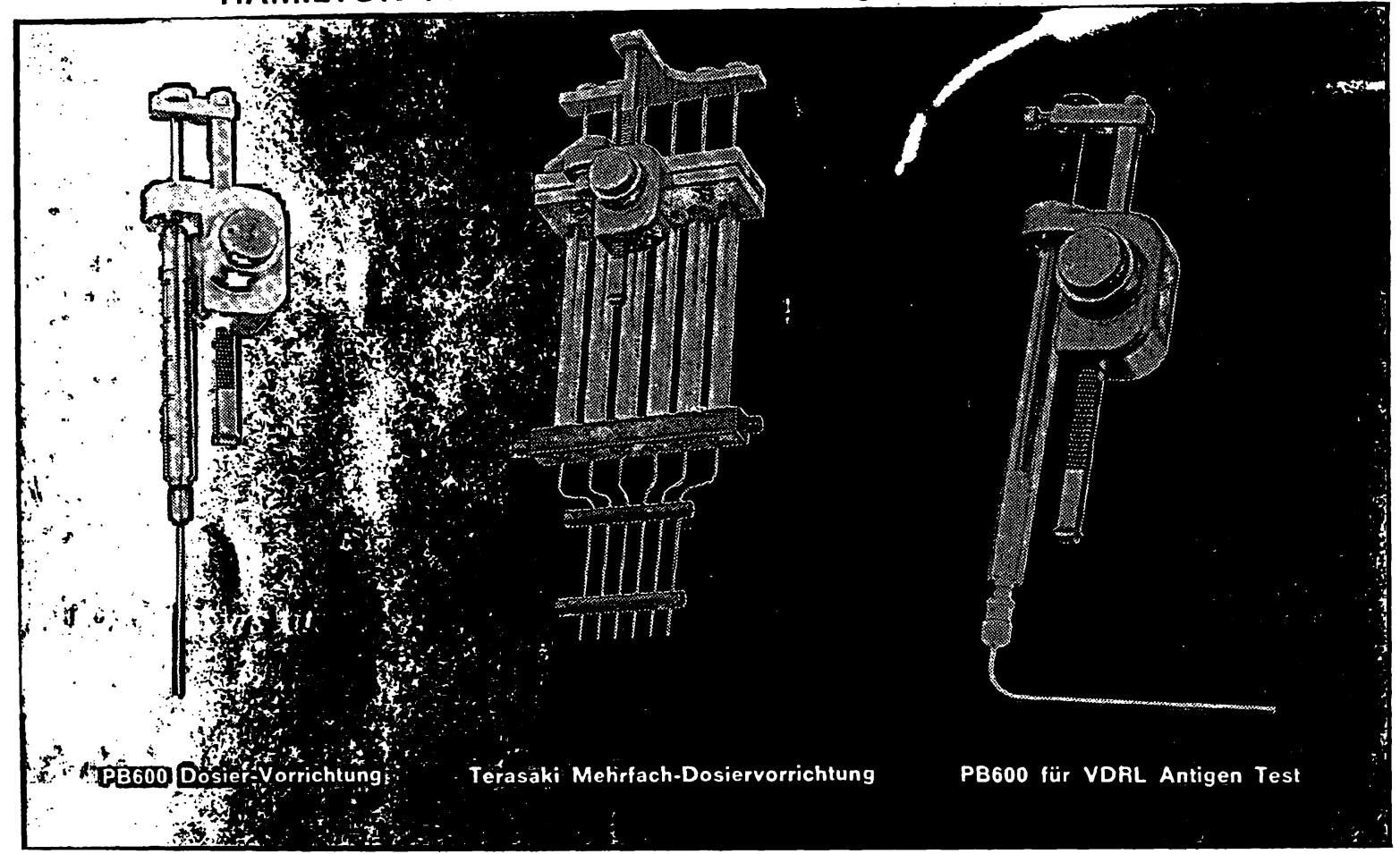

HAMILTON-DOSIERVORRICHTUNGEN GEBEN STETS EINE REPRODUZIERBARE GENAUIGKEIT.

Diese Geräte werden besonders in der Forschung für medizinische und chemische Arbeiten verwendet. Bei Druckknopf-Betätigung wird 1/50 des Spritzenvolumens abgegeben.

Einige von vielen Anwendungsgebieten sind die Dünnschicht-Chromatographie (PB 600), Organtransplantation für die Mikrotechnik der Serumtypisierung in der Humanmedizin (Terasaki). und Serologie für VDRL-Teste. Fordern Sie bitte den neuen Hamilton-Katalog an.

Autorislerter Handler für die BRD:

\section{GUNTHER SGHRIDT}

2 HAMBURG 68 - POSTFACH 680104 - TEL. $6020780 / 6020143$

Bezirksvertretungen:

Aachen: Fa. Ludwig Mohren KG

Berlin: Fa. H. Windler KG

Bonn:

F. C. Gerhardt

Fa. H. Würgens \& Co.
Fa. Willi Fischer KG

Freiburg:

Göttingen:

Kannover:

Karls?

Münchèn:

Münster:
Fa. Bender \& Hobein

Fa. Bodo Schmidt

Fa. Bender \& Hobein

Fa. Erich Eydam

Fa. Schultheiss
Fa. H. Jürgens \& Co.
Generalagentur für Europa:

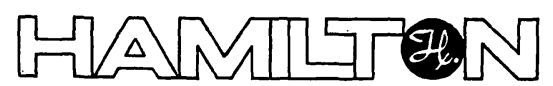

MICROMESURE N.V. 


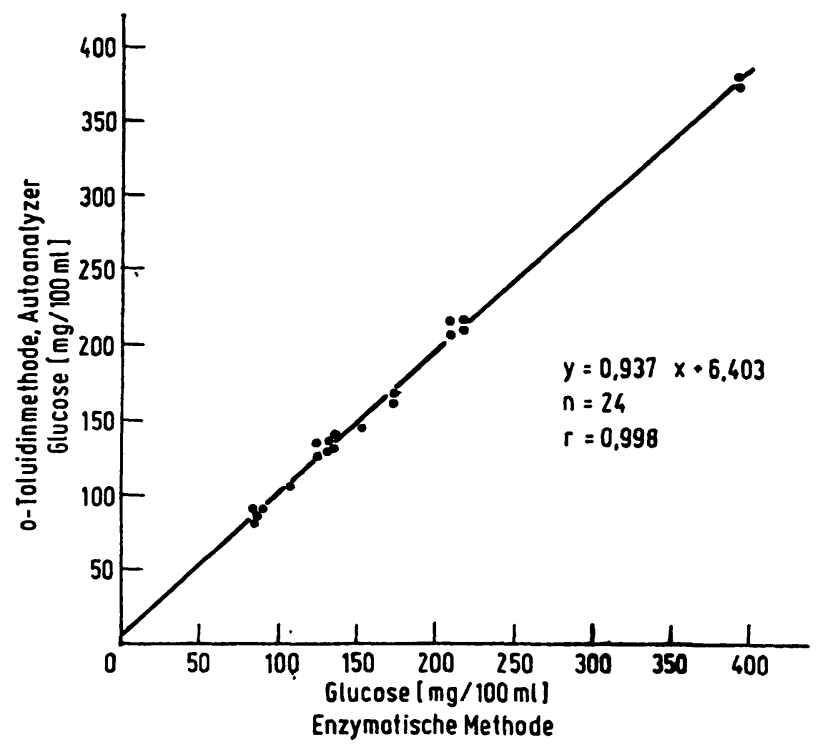

Abb. 3

Darstellung der Ausgleichsgraden der Blutglucosebestimmungen nach Abnahme mit der $20 \mu l$ Mikrokapillare und der $100 \mu l$ Blutzucker pipette (o-Toluidin-Methode bzw. Hexokinase-Glucose-6-phosphatDepydrogenase-Methode)

Das beschriebene Verfahren der Kapillarblutentnahme mit Hilfe von Mikrokapillaren zur Bestimmung der Glucose- bzw. Hämoglobinkonzentration im Kapillarblut ermöglicht ein sauberes, schnelles Arbeiten auch des Ungeübten. Die Präzision dieser Technik steht in keiner Weise den bisher üblichen Verfahren nach. Vielmehr lassen sich mit diesem Verfahren die auf falscher Entnahmetechnik beruhenden Bestimmungsfehler wesentlich reduzieren. Besonders vorteilhaft wirkt sich das kleine Probevolumen für die Blutglucosebestimmung aus, da es in jedem Fall ohne Schwierigkeiten gelingt, präzis diese Menge aus dem Kapillarbluttropfen zu entnehmen. Bei Verwendung des beschriebenen Fließsystems im Autoanalyzer oder der enzymatischen Blut-

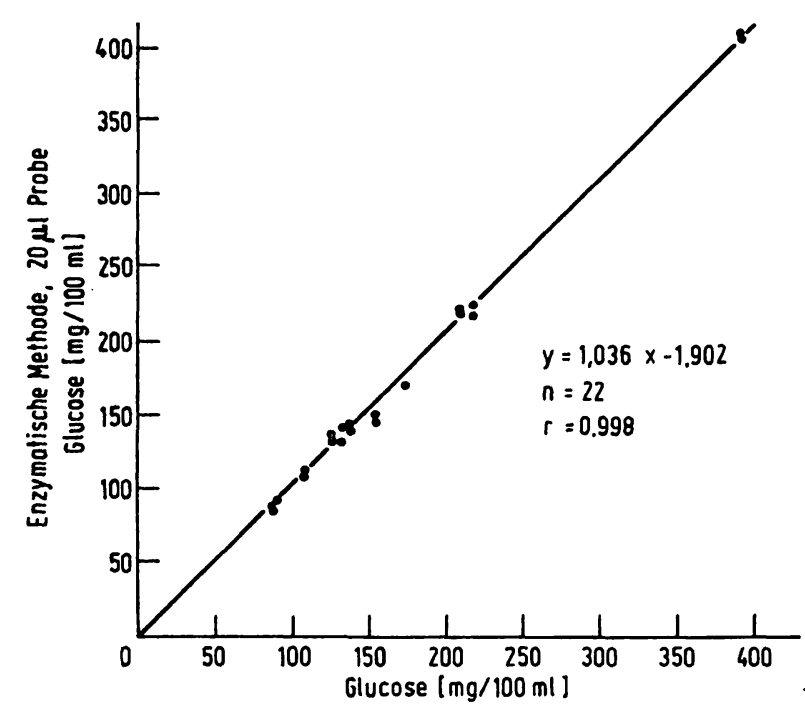

Enzymatische Methode, $100 \mu l$ Probe

Abb. 4

Darstellung der Ausgleichsgraden der enzymatischen Blutglucosebestimmungsmethoden nach Blutentnahme durch $20 \mu l$ Mikrokapillare bzw. $100 \mu l$ Blutzuckerpipette

glucose-Bestimmungsmethode kann selbst bei dieser kleinen Menge mindestens zweimal aus der gleichen Probe eine Bestimmung durchgeführt werden. Die Möglichkeit, das angegebene Entnahmeverfahren sowohl für die enzymatische Blutglucose-Konzentrationsbestimmung wie für die o-Toluidin-Methode im Autoanalyzer und im manuellen Verfahren zu verwenden, erweist sich besonders günstig für die Laboratorien, die im Tag- und Nachtdienst unterschiedliche Bestimmungsverfahren anwenden. Nicht zuletzt wird eine Rationalisierung im Laboratorium durch erhebliche Zeitersparnis sowohl bei der Blutentnahme selbst wie bei der weiteren Analyse und der Reinigung der Glaswaren erzielt.

\section{Literatur}

1. von BoroviczénY, K. G., Erythrocytometric Methods and their Standardization, Verlag S. Karger, Basel (1964). - 2. VAN KAMPEN, E. J. und W. G. Zijustra, Clin. Chim. Acta, Amsterdam 6,
358 (1961). - 3. Hărtel, A., R. Helger und H. Lang, diese Z. 7 , 14 (1969). - 4. Barthelmai, W. und R. Czok, Klin. Wschr. 40, $585,1962$.
Prof. Dr. A. Delbrück 3000 Hannover Podbielskistr. 380 